Accepted for publication, Journal of Behavioral Medicine, May 29, 2018.

\title{
Self-Compassion, Chronic Age-Related Stressors, and Diurnal Cortisol Secretion in Older
}

\section{Adulthood}

\author{
Herriot, Heather ${ }^{1}$, M.A., Wrosch, Carsten ${ }^{{ }^{*}}$, Ph.D., \& Gouin, Jean-Philippe ${ }^{1}$, Ph.D. \\ ${ }^{1}$ Concordia University, Montreal
}

Abstract: 180 words; Introduction: 1527 words; Discussion: 1862 words; Total 6248 words

3 Tables; 1 Figure

Funding: The study has been supported by awards and grants from Canadian Institutes of Health Research to Carsten Wrosch and Jean-Philippe Gouin, and a doctoral fellowship from Social Sciences and Humanities Research Council of Canada to Heather Herriot.

Conflict of Interest: The authors declare that they have no conflict of interest.

Correspondence concerning this article should be addressed to Carsten Wrosch, Concordia University, Department of Psychology and Centre for Research in Human Development, 7141 Sherbrooke Street West, Montreal, QC, H4B 1R6, Canada; e-mail: carsten.wrosch@concordia.ca. 


\begin{abstract}
Background: Many older adults experience chronic age-related stressors (e.g., life regrets or health problems) that are difficult to control and can disturb cortisol regulation. Self-compassion may buffer adverse effects of these stressful experiences on diurnal cortisol secretion in older adulthood.

Purpose: To examine whether self-compassion could benefit older adults' cortisol secretion in the context of chronic and largely uncontrollable age-related stressors. Methods: 233 communitydwelling older adults reported their levels of self-compassion, age-related stressors (regret intensity, physical health problems, and functional disability), and relevant covariates. Diurnal cortisol was measured over 3 days and the average area-under-the-curve (AUC) and slope were calculated. Results: Higher levels of self-compassion were associated with lower daily cortisol levels among older adults who reported higher levels of regret intensity, physical health problems, or functional disability $(\beta s<-.54, p \mathrm{~s}<.01)$, but not among their counterparts who reported lower levels of these age-related stressors $(\beta \mathrm{s}<.24, p \mathrm{~s}>.28)$. Conclusions: These results suggest that selfcompassion may represent an important personal resource that could protect older adults from stressrelated biological disturbances resulting from chronic and uncontrollable stressors.
\end{abstract}

Keywords: aging; cortisol; self-compassion; stressors 


\section{Introduction}

The aging population represents the fastest growing segment of the human population, typically including individuals of 60 years of age and older (United Nations, 2015). Although normative aging involves both developmental gains and losses (Baltes, 1987), research has documented an age-related increase in the amount of chronic and less controllable stressors (e.g., physical health problems, functional disabilities, or life regrets; Heckhausen et al., 2010; Wrosch et al., 2006; Wrosch et al., 2007; Ebner, Freund, \& Baltes, 2006; Heckhausen, Dixon, \& Baltes, 1989). These stress experiences may trigger psychological distress and forecast disturbances in HPA axis regulation leading to altered patterns of cortisol release (Cohen et al., 2007; Miller, Chen, \& Zhou, 2007). As such, examining patterns of cortisol dysregulation may be particularly important in older adulthood because HPA axis regulation can become compromised (Sapolsky, Krey, \& McEwen, 1986) and the controllability of certain stressors frequently decrease during this life phase (Ebner et al., 2006; Heckhausen et al., 2010). As a result, cortisol levels can be higher in older adulthood (Nater et al., 2013), and these cortisol disturbances (e.g., higher areaunder-the-curve [AUC] or a flatter cortisol slope) may reflect dysregulated physiological responses to stress experiences (Nater et al., 2013; Nicolson et al., 1997; Otte et al., 2005; Rohleder et al., 2002). Such patterns of cortisol dysregulation are thought to have important implications for health over time (Heim et al., 2000; Sephton et al., 2000) and have been associated with higher mortality rates in middle-aged and older adults (e.g., Kumari et al., 2011).

Although the general link between stress and health-relevant biological processes has been documented (Cohen et al., 2007), the effects of some stressors on health-related outcomes may become paramount among older adults. In particular, chronic and uncontrollable stressors have been shown to adversely affect HPA axis activity, as they tend to be associated with higher levels and flatter slopes of cortisol outflow (Miller, Chen, \& Zhou, 2007). As older adults are 
likely to experience an increase in these types of stressors (Ebner, Freund, \& Baltes, 2006; Heckhausen, Dixon, \& Baltes, 1989), their biological stress response may also be affected. Consistent with this argument, up to $90 \%$ of older adults report significant life regrets that are perceived as less controllable than young adults' life regrets (Landman, 1987; Wrosch \& Heckhausen, 2002). In addition, intense regret experiences have been associated with psychological distress and enhanced cortisol output (Wrosch et al., 2005; 2007). Such adverse effects of life regrets may occur in old age because the opportunities for undoing the consequences of regretted events often become sharply reduced or absent as individuals advance in age (Wrosch et al., 2005). Thus, older adults who experience intense regret may be at risk of ruminating about the regretted event without being capable of overcoming the regret, which may trigger greater distress and a disturbance of HPA axis regulation (Heckhausen, Wrosch, \& Schulz, in press; Wrosch \& Heckhausen, 2002).

Older adulthood is also characterized by other, frequently intractable, stressful experiences, such as an increase in physical health problems and functional disability. Indeed, both of these stressors have been related with psychological distress (e.g., Bruce, 2001) and dysregulated cortisol secretion (Heaney et al., 2012; Wrosch et al., 2007). Such effects may occur in older adulthood since a loss of control over the onset and progression of physical health problems can be psychologically and physiologically distressing (Heckhausen, Wrosch, \& Schulz, in press).

It is important to note, however, that chronic and uncontrollable stressors may not disturb cortisol secretion to the same extent among all individuals (Wrosch et al., 2007). Whether stressful experiences result in dysregulated HPA activity can vary considerably between individuals (Kudielka et al., 2009). Such individual difference variables can be partially independent from the experience of a stressor and determine how stressors impact important 
outcomes (Bolger \& Zuckerman, 1995). In this regard, past research has shown that factors such as social support and physical activity can exert robust stress-buffering effects (e.g., Rimmele et al., 2007; Seeman et al., 1994). Similarly, research suggests that inter-individual differences in psychological characteristics that facilitate adjustment to, and coping with, stress are likely to moderate links between stress and cortisol (e.g., O’Donnell et al., 2008; Wrosch et al., 2007). Thus, psychological characteristics that facilitate coping with stressors are likely to buffer the adverse effects that these stress experiences can exert on older adults' biological functioning (Heckhausen et al., 2010).

To determine individual difference variables that may promote effective coping with chronic and uncontrollable stressors in old age, motivational theories of life-span development provide a useful framework for identifying pathways to successful aging (e.g., Brandtstädter \& Renner, 1990; Heckhausen et al., 2010, in press). These theories posit that older adults' health may benefit from a shift towards self-regulation processes that involve self-protective strategies, helping them to accept that certain problems can no longer be resolved (e.g., social comparisons or positive reappraisals; Wrosch et al., 2006). Building upon these theories, we propose that selfcompassion could represent a dispositional factor that exerts such motivational function and promotes older adults' physiological regulation in the context of uncontrollable stress experiences. Self-compassion is an individual difference variable that involves treating oneself in a manner that most individuals would treat a close friend who experiences difficult life circumstances. That is, with kindness and concern, rather than self-criticism, pity, or aggrandizing negative feelings (Neff, 2003a). Self-compassionate individuals are supportive and understanding towards themselves and maintain an open and non-judgmental attitude of oneself during difficult times. In addition, they are able to recognize that difficult life circumstances are common to the human experience (Neff, 2003b). 
As a consequence, we suggest that individual differences in dispositional self-compassion may prevent biological disturbances in response to uncontrollable stressors by promoting psychological processes that reduce emotional distress (cf. Cohen et al., 2007). In circumstances when older adults experience difficulty performing certain behaviors (e.g., living independently) or have severe life regrets, some of them may engage in self-blame or self-criticism and keep trying to resolve stress experiences that are difficult or impossible to overcome (Wrosch et al., 2005). By contrast, self-compassionate individuals should be less likely to engage in such maladaptive psychological responses to encountering uncontrollable problems (Brion et al., 2014; Leary et al., 2007; Neff, 2011) and instead may be more likely to accept that their health is declining or forgive themselves for a behavior that they regret (Zhang \& Chen, 2016). These motivational concomitants of self-compassion may ameliorate the psychological consequences of uncontrollable stress experiences and reduce associated cortisol output.

In support of these possibilities, research has documented that self-compassion can moderate the effects of age-related stressors, such as poor physical health, pain, and mobility on psychological well-being (Allen et al., 2012; Homan, 2016). Moreover, self-compassion can promote physical health among individuals who confront chronic disease and experience higher levels of distress (e.g., diabetes, Friis et al., 2015). Less is known, however, about whether selfcompassion also benefits physiological functioning in the context of stressful experiences. Preliminary evidence from laboratory stress tasks among young adults suggests that selfcompassion could promote more adaptive biological responses to stress, such as reduced cortisol reactivity and inflammation (Breines et al., 2014a; 2014b). However, research examining the effects of self-compassion on diurnal cortisol output in the context of older adults' naturalistic, self-relevant, and uncontrollable stress experiences is lacking. We think that such research may be warranted since associations between stress and biological dysregulation can become 
paramount in older adulthood (Kiecolt-Glaser \& Glaser, 2001). In addition, it could help identify a psychological mechanism that influences HPA functioning among older adults who experience common chronic and uncontrollable age-related stressors (Adam et al., 2007; Van Eck et al., 1996).

\section{The Present Study}

The present study examined whether individual differences in dispositional selfcompassion predict lower levels of diurnal cortisol output among older adults who experience chronic and uncontrollable age-related stressors. Using cross-sectional data from 233 older adults, we examined associations among self-compassion, specific age-related stressors (physical health problems, functional disability, and regret intensity), and diurnal cortisol secretion.

Because overall daily cortisol secretion (i.e., higher AUC) and change in cortisol secretion across a day (i.e., flattened slope) have both been associated with stress experiences, morbidity, and mortality, we predicted these indicators in our analyses (Heim, et al., 2000, Kumari et al., 2011; Sephton et al., 2000). In supplemental analyses, we further explored whether obtained effects were related to cortisol output at different times of day (i.e., awakening, CAR, afternoon, and evening). More specifically, we expected that higher levels of age-related stressors (physical health problems, functional disability, and regret emotions) could be associated with higher daily levels and a flattened slope of cortisol secretion. In this regard, we hypothesized that the emergence of such an association would depend on older adults' levels of self-compassion. In particular, we predicted that the experiences of age-related stressors would exert a significant effect on cortisol secretion only among older adults who score low on self-compassion. By contrast, we hypothesized that their counterparts who are more self-compassionate would be protected from experiencing cortisol disturbances in context of higher levels of age-related stressors. To examine potential confounds of the observed effects, the analyses controlled for 
socio-demographic characteristics (age, sex, and socio-economic status) and health relevant covariates (BMI, smoking status).

\section{Methods}

\section{Participants}

Participants in this study included an age-normative sample of community-dwelling older adults who participated in the Montreal Aging and Health Study (MAHS). The MAHS is a longitudinal study that originally included 215 participants (Wrosch et al., 2007). After ten years of study, the cohort of the MAHS was refreshed and new measures that are pertinent for the present study (e.g., self-compassion) were added to the study. Therefore, only cross-sectional data from this time point were analyzed. Recruitment for this study was completed via newspaper advertisements in the Montreal area. To obtain a normative sample, the only inclusion criteria for this study was that participants were older than 60 years. Each participant provided informed consent prior to participating in the study.

In this refreshed cohort, a total of 268 participants (95 original and 173 newly recruited participants) were assessed in their homes or in the laboratory. Participants were excluded from the analysis if they did not provide at least one complete day of cortisol data $(n=30)$. Five additional participants were excluded for not completing the self-compassion scale. The analytic sample thus included 233 participants. Excluded participants had a significantly higher BMI (M $=28.83, S D=5.28)$ than included participants $(M=26.80, S D=4.78 ; t(258)=-2.24, p<.03$. The excluded participants did not significantly differ on any of the other main study variables $(|t \mathrm{~s}|<1.28, p \mathrm{~s}>.20)$

\section{Materials}

Diurnal Cortisol. To assess normative patterns of diurnal cortisol secretion, we measured cortisol on three non-consecutive days over the course of one week. Participants were 
asked to collect five saliva samples using salivettes at awakening, 30 minutes after awakening, 2 PM, 4 PM, and bedtime. Upon awakening participants collected the first sample and started a timer to facilitate the collection of the second saliva sample thirty minutes after awakening. Participants were then contacted by phone at 2 PM and 4 PM to facilitate compliance with the afternoon samples collection. The last sample of the day was collected by the participants themselves before they went to bed. The time each sample was collected was recorded by the participants. To prevent contamination with food or blood, they were instructed not to brush their teeth or eat prior to saliva collection. For each sample, participants were instructed to insert a salivette into their mouths for 30 seconds to collect saliva. The salivettes were stored in participants' home refrigerators until they were returned to laboratory (after 2-3 days) and frozen until study completion. Cortisol analysis was performed at the University of Trier using a timeresolved fluorescence immunoassay with a cortisol-biotin conjugate as a tracer. The inter-assay variability from these cortisol analyses performed at the University of Trier was on average 5.3\% and the intra-assay variability performed in this laboratory is usually below $10 \%$.

All cortisol scores were log-transformed to stabilize variance. Cortisol samples were excluded if they deviated three standard deviations or more from the mean cortisol level for a given time of day, as these samples could have been contaminated with food or blood. Cortisol scores were only calculated for participants who provided at least four usable cortisol scores on each of the collection days. Daily cortisol levels were calculated using the area-under-the-curve with respect to ground (AUC) across each day separately, using the trapezoidal method based on hours after awakening (Pruessner et al., 2003). The 30-minutes measure was excluded from the calculation of daily cortisol level because early morning increase of cortisol has been shown to be relatively independent from overall cortisol level (Chida \& Steptoe, 2009). The three AUC scores were averaged across the three days to obtain a reliable measure of average cortisol 
secretion. Cortisol slope was calculated by regressing cortisol values on time of day for each collection day (excluding the 30-minute measure). The three obtained cortisol slopes were then averaged to create a reliable measure of average cortisol slope. On each of the assessment days, cortisol levels significantly declined from awakening to bedtime, $t \mathrm{~s}>26.44, p \mathrm{~s}<.001$.

Self-Compassion was measured with the 12-item Self-Compassion Scale (Raes et al., 2011). This short-form version of the Self-Compassion Scale has shown good internal consistency $(\alpha \mathrm{s} \geq .86)$, good test-retest reliability over 5 months $(\alpha=.71$; Neff, 2003a), a high correlation with the long-form scale $(r \mathrm{~s}>.97)$, and self-compassion interventions have been shown to result in increases of self-compassion scores (Neff \& Germer, 2013; Raes, 2011; Raes et al., 2011). This questionnaire uses 5-point Likert-type scales (almost never $=0$ to almost always $=4$ ). Participants were asked to consider how they typically act towards themselves in difficult times. Samples items include: "I try to see my failings as part of the human condition", "When I fail at something important to me, I become consumed by feelings of inadequacy", and "I try to be understanding and patient towards those aspects of my personality I don't like." To obtain an indicator of self-compassion a sum score of the 12 items was calculated after negatively formulated items were reverse coded ( $\alpha=.80$ in the current sample).

Physical health problems were measured using a previously used symptom checklist of seven physical health problems (Wrosch et al., 2005). This checklist asked participants to report whether they had experienced or had been treated for any of the following health problems in the past 12 months: (a) persistent skin troubles (e.g., eczema); (b) recurring stomach trouble, indigestion, or diarrhea; (c) being constipated all or most of the time; (d) chronic sleeping problems; (e) migraine headaches; (f) asthma, bronchitis, or emphysema; and (g) thyroid disease. To obtain an indicator of physical health problems, we computed a count variable of each of these seven health problems (Range $=0-5, M=1.24, S D=1.12$ ). 
Functional disability was measured by asking participants to indicate whether or not they had difficulty or were unable to perform six basic activities of daily living (eating, using the toilet, dressing, showering, walking around the home, and getting in and out of a bed or chair), and six instrumental ADLs (heavy housework, light housework, shopping, preparing meals, managing money, and using the phone; Lawton \& Brody, 1969). A count variable was computed comprising of the total number of basic and instrumental ADL difficulties (Range $=0-9, M=$ $1.71, S D=2.20)$

Regret intensity. Participants were asked to think about their own lives and report their most severe life regret. Consistent with past research, the majority of the sample reported having a significant life regret (82\%; Bauer et al., 2008; Landman, 1987). To determine regret intensity, participants were asked to report the extent to which they experienced the following emotions during the past few months when they thought about their life regrets: sorrow, anger, desperate, irritated, helpless, and embarrassed (based on the work by Gilovich et al., 1998). Each item was rated on a 5-point Likert-type scale (not at all $=0$ to extremely $=4$ ). An index of the regret intensity was calculated by computing a sum score of the 6 items $(\alpha=.85)$. Individuals who reported not having a life regret and did not complete the regret intensity scale $(\mathrm{n}=18)$ received a score of zero for this construct.

Covariates included participants' sex, age, objectively measured BMI, smoking status (yes or no), and SES. These variables were selected as covariates because of their previously demonstrated associations with cortisol secretion (e.g., Hajat et al., 2010; Van Cauter et al., 1996). Sex was coded as male $=1$ and female $=2$. SES was indexed using three variables; highest education completed, yearly family income, and perceived social status. The three standardized SES measures were correlated $(r \mathrm{~s}=.36$ to $.50, p \mathrm{~s}<.01)$ and averaged to obtain a reliable indicator of SES. 


\section{Data Analysis}

Preliminary analyses were conducted to describe the sample (means, standard deviations, and frequencies) and explore zero-order correlations among the main study variables. The main hypotheses of this study were tested using multiple regression analysis (SPSS 23.0). A total of 9 participants had missing data on regret emotions, and 6 participants did not provide information necessary to calculate BMI. Since the proportion of missing data was less than $5 \%$, missing scores of single constructs were replaced with the sample mean in the regression analysis (Tabachnik et al., 2013). All predictor variables were standardized prior to conducting the main analysis. In the first step of the analysis all covariates and main effects (sex, age, BMI, smoking status, SES, self-compassion, regret emotions, and physical health problems) were included in the analysis. The final step involved testing the hypothesized interaction effects in separate models (physical health problems x self-compassion, functional disability x self-compassion, and regret emotions x self-compassion). Significant interactions were plotted to illustrate the associations between self-compassion and cortisol levels $1 S D$ above, average, and $1 S D$ below the sample mean of physical health problems, functional disability and regret intensity, and the simple slopes were tested for significance (Aiken \& West, 1991).

Finally, we conducted supplemental analyses to explore whether obtained, significant effects of chronic stressors on cortisol AUC and slope were related to specific times of day by predicting in separate analyses awakening levels, awakening response (CAR; sample 1 subtracted from sample 2), afternoon levels (averaged samples 3 and 4), and evening levels of cortisol as outcome variables.

\section{Results}

\section{Preliminary Analyses}

Sample characteristics are reported in Table 1. Participants were on average 76 years old 
$($ Median $=76$, Range $=59-93)$. Approximately 39\% of the sample was male. This sample represented a diverse socioeconomic background, approximately $33 \%$ had an income less than $\$ 34,000,40 \%$ had an income between $\$ 34,001$ and $\$ 85,000$, and $16 \%$ had an income greater than $\$ 85,000$. Participants reported a perceived social status that was slightly above mid-range. More than half the sample had a BMI greater than 25. A minority of participants smoked (5\%).

Zero-order correlations among the study variables are reported in Table 2. Cortisol levels (AUC) were negatively associated with self-compassion and positively associated with age. Males were more likely to secrete higher cortisol levels than females. Cortisol slope was also positively associated with age. Self-compassion was negatively associated with regret intensity and positively associated with socioeconomic status. Regret intensity was negatively associated with socioeconomic status. Functional disability was positively associated with physical health problems and age and negatively associated with SES. Females reported higher levels of functional disability and physical health problems than males.

\section{Predicting Diurnal Cortisol Level}

The results of the regression analysis predicting participants' cortisol levels (AUC) are reported in Table 3 . The first step of the analysis, which included all covariates and main study variables, showed a significant model effect $F(9,232)=3.15, p<.01$. Age was significantly positively associated with cortisol levels $\beta=.40, p=.02$. Sex was significantly negatively associated with cortisol levels $\beta=-.54, p<.01$, indicating that females had lower cortisol levels than males. No significant main effects of the remaining covariates or self-compassion and agerelated stressors (physical health problems, functional disability, and regret emotions), were obtained for predicting participants' cortisol levels. In the final step of the analysis, three significant interaction effects emerged between self-compassion and the three age-related stressors (physical health problems: $\beta=-.52, p<.01$, functional disability: $\beta=-.29, p \leq .05$, and 
regret emotions: $\beta=-.44, p<.03)$.

The significant interaction between self-compassion (1 SD above and below the sample mean) and physical health problems is plotted in the upper panel of Figure 1, separately for participants who reported low (-1 SD), average (sample mean), and high (+1 SD) levels of physical health problems. The observed pattern suggests that particularly high levels of cortisol were observed among participants who reported high levels of physical health problems and low levels of self-compassion. In addition, it indicates that to the extent participants' reported greater levels of physical health problems, self-compassion became increasingly associated with lower levels of daily cortisol. Follow-up simple slope analyses supported these interpretations. Selfcompassion significantly predicted lower cortisol levels among participants who reported high levels of physical health problems $\beta=-.79, S E=.22 t=-3.54, p<.01$, but not among their counterparts who reported either average, $\beta=-.27, S E=.17, t=-1.68, p=.10$, or low levels of physical health problems $\beta=.24, S E=.22, t=1.08, p=.28$.

The significant interaction effect between self-compassion and functional disability is plotted in the middle panel of Figure 1, separately for participants who reported low $(-1 S D)$, average (sample mean), and high $(+1 S D)$ levels of functional disability. Similar to the previous interaction, the obtained pattern shows that relatively high levels of cortisol were observed among participants who reported high levels of functional disability and low levels of selfcompassion. The results further suggest that to the extent participants' reported higher levels of functional disability, self-compassion became increasingly associated with lower levels of cortisol levels. Simple slope analysis were consistent with this interpretation, indicating that among those participants who report higher levels of functional disability, self-compassion became more strongly associated with lower cortisol levels $\beta=-.54, S E=.22, t=-2.48, p=.01$, as compared to their counterparts who had either average $\beta=-.24, S E=.17, t=-1.44, p=.15$ or 
low levels of functional disability $\beta=.05, S E=.24, t=.21, p=.83$.

The significant interaction effect between self-compassion and regret intensity is plotted in the lower panel of Figure 1, separately for participants who reported low (-1 SD), average (sample mean), and high $(+1 S D)$ levels of regret intensity. Similar to the previously reported interactions, the obtained pattern suggests that the highest cortisol levels appeared among those who experienced high levels of regret intensity and were relatively low in self-compassion. Selfcompassion was increasingly associated with lower cortisol levels to the extent that participants experienced higher levels of regret intensity. This interpretation was supported by the simple slope analyses, demonstrating that higher self-compassion significantly predicted lower cortisol levels among participants who reported greater levels of regret intensity, $\beta=-.67, S E=.25, t=-$ $2.71, p<.01$, but not among their counterparts who reported either average, $\beta=-.23, S E=.21, t$ $=-1.10, p=.27$, or low levels of regret intensity, $\beta=.09, S E=.23, t=.39, p=.70$.

The supplemental analyses, predicting different times of cortisol secretion during the day, showed that the obtained interaction between physical health problems and self-compassion predicted awakening levels, CAR, and evening levels of $\left(|\beta| \mathrm{s}>|.02|, p \mathrm{~s}<.05, R^{2}>.02\right)$, but not afternoon levels of cortisol $\left(\beta=-.02, p=.11, R^{2}=.01\right)$. In addition, the interaction between regret intensity and self-compassion predicted only evening levels $\left(\beta=-.05 p=.001, R^{2}=.04\right)$, but not awakening levels, CAR, or afternoon levels of cortisol $\left(|\beta| \mathrm{s}<|-.02|, p \mathrm{~s}>.22, R^{2}<.01\right)$. Finally, the interaction between functional disability and self-compassion did not significantly predict any additional marker of cortisol $\left(|\beta| \mathrm{s}<|-.02|, p \mathrm{~s}>.16, R^{2}<.01\right)$.

\section{Predicting Cortisol Slope}

The results of the regression analysis predicting cortisol slope are also reported in Table 3. The first step of the analysis, which included all covariates and main study variables, showed a significant model effect $F(9,232)=2.06, p<.04$. Of the covariates, age was significantly 
positively associated with cortisol slope, $\beta=.003, p=.01$, indicating that older, as compared to younger, participants exhibited a more flattened cortisol slope across day. However, no additional significant associations with cortisol slope were obtained for the remaining covariates, self-compassion, or age-related stressors (physical health problems, functional disability, and regret emotions). The final step of the analysis, testing the three interaction effects between selfcompassion and age-related stressors separately (physical health problems, regret emotions and functional disability), showed that none of the three interactions significantly predicted participants' cortisol slope, all $|\beta s|<.002$, all $p s>.09$ (see Table 3). ${ }^{1}$

\section{Discussion}

This study showed in a community-dwelling sample of older adults that dispositional self-compassion moderates the associations between specific chronic and uncontrollable agerelated stressors (i.e., physical health problems, functional disability, and regret intensity) and higher levels of diurnal cortisol secretion. More specifically, we found that to the extent older adults reported higher levels of regret and health-related stressors, self-compassion became increasingly associated with lower levels of diurnal cortisol (AUC). These findings were not observed for participants' cortisol slope, and the obtained pattern of results was significant after controlling for a number of demographic (age, sex, SES) and health-relevant covariates (BMI, smoking).

The study's findings suggest that self-compassion represents an adaptive personal resource that is capable of buffering stress-related disturbances of older adults' cortisol secretion. These findings are consistent with motivational theories of life-span development, which document that successful aging is characterized by a shift in older adults' self-regulation attempts, from overcoming problems and striving for gains to adjusting psychologically to relatively intractable age-related losses (Brandtstädter, \& Renner, 1990; Heckhausen et al., 2010, 
in press). In fact, certain self-protective processes (e.g., avoiding self-blame, positive reappraisals, or downward social comparisons) and goal disengagement processes (Heckhausen et al., 2010; Wrosch et al., 2006) have been shown to exert effective coping effects in the context of older adults' stress experiences. To this end, self-compassion may foster such self-protective responses to the experience of uncontrollable stressors. For example, being kind and understanding to oneself during times of stress could promote positive reappraisals of difficult life circumstances and reduce self-blame for emerging problems, rumination, or catastrophizing (Brion et al., 2014; Leary et al., 2007; Neff, 2011). In addition, appraising personal challenges in the broader context of common humanity may elicit adaptive social comparisons that may reduce negative emotional responses to pressing problems (Festinger, 1954) and prevent the HPA axis from releasing high levels of cortisol into the circulation.

Of importance, the beneficial effects of self-compassion on older adults' cortisol secretions were observed in the presence of both psychological stressors (i.e., intense life regret) and physical stressors (i.e., functional disability and physical health problem). These results suggest that self-compassion may represent a dispositional resource that can protect older adults' cortisol functioning across a variety of life stressors. Since both intense life regrets and physical health problems typically represent relatively chronic stressors that become increasingly intractable as individuals advance in age (Wrosch et al., 2005; 2006), these findings may further imply that self-compassion is adaptive particularly if individuals confront uncontrollable and chronic stressors. To this end, we note that our study also included a measure of daily perceived stress, which is thought to be different from chronic stress experiences (Almeida, 2005), and could be elicited by both stressors that are either transient and potentially controllable or by chronic and intractable stressors. Interestingly, self-compassion did not interact with daily perceptions of stress in predicting cortisol output (see Footnote 1), which could imply that 
participants' daily stress experiences were relatively minor and/or potentially controllable. As a consequence, the influence of self-compassion on older adults' cortisol secretion could be stronger in the context of chronic and intractable stressors, as compared to daily perceptions of stress.

It should be noted that consistent moderation effects of self-compassion in the associations between age-related stressors and cortisol were found only for predicting cortisol AUC. By contrast, the analyses did not show the same effects for predicting cortisol slope. In addition, the supplemental analyses indicated that if cortisol secretion during different times of day were analyzed separately, buffering effects of self-compassion were either absent (i.e. for functional disability) or detected only for some times of the day (i.e., for regret experiences and physical health problems). These findings may imply that cortisol AUC is a particular promising outcome measure, which is consistent with some past research that has documented associations between stress experiences and cortisol AUC only (Ice, 2005; Wrosch et al., 2005). Such different effects may occur considering that stress experiences can enhance cortisol secretion across the entire day and thus would be particularly likely to predict cortisol AUC (Kirschbaum et al., 1995). Cortisol measures that reflect different times of day, by contrast, may fail in capturing some of the physiological effects of stressors occurring during the entire day. In addition, a flattened slope can be observed as a function of both reduced morning cortisol levels and/or greater levels of evening cortisol, and thus may be less related to stressors that increase cortisol across the entire day (Cohen et al., 2006). Another explanation that is consistent with our data (see correlations in Table 2) would be that cortisol AUC and slope can be relatively independent from each other (Vedhara et al., 2006) and may be regulated by different mechanisms (Ice, 2005). This possibility could further explain the observed associations between self-compassion and cortisol AUC among individuals with low self-compassion, considering 
previous research documenting that cortisol level may be more affected by psychological traits, whereas cortisol slope may be influenced to a larger extent by variables related to the circadian rhythm (i.e., age, physical activity, sleep; Ice, 2005). We further acknowledge that the reliabilities of cortisol slope and levels during different times of day may not have been sufficiently high to detect between-person differences. Although previous research has shown that three collection days provide reliable estimates of between-person differences in cortisol level, more collection days are likely required to obtain other reliable indicators of cortisol secretion (e.g., Segerstrom et al., 2014).

Finally, we would like to acknowledge that our data did not show significant main effects of any of the age-related stressors on participants' cortisol secretion. This pattern is consistent with prominent self-regulation theories that posit that not stressors per se, but instead individuals' appraisals and behaviors, determine the health-related outcomes of stress experiences (Folkman et al., 1986). In support of this possibility, we note that other studies also did not consistently report significant main effects of stress experiences on cortisol secretion (e.g., Kudielka et al., 2009; Liu et al., 2014). As such, our data suggest that individual difference variables that facilitate adjustment to stressors, such as self-compassion, may play an important role in the physiological effects of stress experiences. In the absence of adaptive levels of selfcompassion, older adults may remain vulnerable and secrete high levels of cortisol in response to intractable stress experiences.

Overall, the reported findings have important implications for research on personality, aging, and cortisol regulation. Our study extends preliminary laboratory-based research (e.g., Breines et al., 2014a) by documenting that differences in dispositional self-compassion can facilitate adaptive stress-related HPA axis responses as older adults go about their normal daily activities. These findings are particularly important given that naturalistic patterns of cortisol 
secretion may provide relevant information for understanding pathways to biological dysregulation and disease (Adam et al., 2007; Van Eck et al., 1996). Indeed, such indicators of dysregulated cortisol secretion have been shown to predict mortality rates among older adults specifically (Kumari et al., 2011).

The obtained findings also contribute to theories of successful aging. With advancing age, individuals typically experience an increasing number of relatively intractable stressors in a variety of life domains, which are likely to compromise their biological functioning and physical health (Wrosch et al., 2006). To this end, our results document that self-compassion buffered the effects of both psychological and physical stressors on increased cortisol output. This pattern of results adds to current motivational theories of life-span development (e.g., Heckhausen et al., 2010) by pointing to the possibility that self-compassion represents an individual difference variable that facilitates effective coping with various age-related stressors across different areas of life.

The reported study has further implications for theory and research on stress and cortisol functioning. Self-compassion buffered the effects of age-related stressors on daily cortisol levels, but not cortisol slope. This mixed pattern may imply that research on psychological factors impacting the human stress response (Ice, 2005; Wrosch et al., 2007) could obtain particularly reliable results by predicting the overall level of cortisol secretion instead of focusing on differentially increasing or decreasing cortisol levels across the day (i.e., slope). In support of this possibility, our findings suggest that cortisol AUC and cortisol slope were not significantly correlated, and future research should investigate more thoroughly the potential mechanisms underlying different associations between stress experiences, psychological factors, and cortisol indicators.

Finally, our findings may have implications for practitioners and clinicians, as they 
highlight the possibility that self-compassion can benefit health-relevant biological functioning in older adulthood. This conclusion points to the utility of self-compassion as a psychological variable that could be fostered in clinical settings to promote physical health among older adults experiencing a variety of age-related stressors. Indeed, specific psychological interventions that foster self-compassion (e.g., Compassion-Focused Therapy, Mindfulness-Based Stress Reduction, Mindfulness-Based Cognitive Therapy, and Acceptance and Commitment Therapy) have been developed, and a recent review of this literature suggests that such interventions can benefit psychological well-being and, in some circumstances, physical health among older adults (Geiger et al., 2016).

There are several limitations of this study that should be addressed in future research. First, while it is a strength of our study to examine associations between self-compassion and cortisol secretion in a naturalistic setting, the analyses were based on a relatively small sample of community-dwelling older adults, and thus may not generalize to the entire aging population. Second, the study used cross-sectional data and thus precludes any causal interpretation. As a consequence, we cannot determine the time-related associations between stressors, selfcompassion, and cortisol output. Third, although our analysis showed convergent associations between self-compassion and stress-related cortisol output for three different stressors (i.e., functional disability, physical health problems, and life regrets), other stress experiences that frequently arise in old age were not assessed in our study (e.g., social losses or support reductions). Fourth, the study did not consider other psychological factors that could be associated with self-compassion and may protect older adults during the experience of agerelated stressors (e.g., control strategies or goal disengagement capacities; Wrosch et al., 2005; 2006). Fifth, our study examined how dispositional variation in self-compassion could buffer the effects of age-related stressors, and did not examine state levels of self-compassion in response 
to specific stressors. Future research should thus further explore whether self-compassion as an active behavioral choice, independent of general individual differences in self-compassion, can also influence the physiological response to specific age-related stressors. Finally, the reported study did not examine clinical health outcomes, and thus could not conclude whether the protective effects of self-compassion on stress-related cortisol disturbances can optimize older adults' physical health over time. Future research should address these limitations by examining larger samples of older adults over an extended period of time. Such research should cover a wider range of age-related stressors, potentially protective psychological factors, and physical health outcomes. Research along these lines may illuminate the psychological and biological mechanisms that enable older adults to manage age-related stressors and protect their quality of life.

\section{Conclusion}

The present study identifies self-compassion as an individual difference variable that is associated with adaptive cortisol functioning in the context of older adults' chronic and uncontrollable stress experiences. The reported results may have implications for clinical interventions that foster self-compassion in older adulthood as a way to cope with common agerelated stressors. 


\section{References}

Adam, E. K., Klimes-Dougan, B., \& Gunnar, M. R. (2007). Social regulation of the adrenocortical response to stress in infants, children, and adolescents. Human Behavior, Learning, and the Developing Brain: Atypical Development, 264-304.

Aiken Leona, S., \& West Stephen, G. (1991). Multiple regression: Testing and interpreting interactions. Sage.

Allen, A. B., Goldwasser, E. R., \& Leary, M. R. (2012). Self-compassion and well-being among older adults. Self \& Identity, 11(4), 428-453. http://dx.doi.org/10.1037/a0014180

Almeida, D. M. (2005). Resilience and vulnerability to daily stressors assessed via diary methods. Current Directions in Psychological Science, 14, 64-68. http://dx.doi.org/10.1111/j.0963-7214.2005.00336.x

Baltes, P. B. (1987). Theoretical propositions of life-span developmental psychology: On the dynamics between growth and decline. Developmental Psychology, 23, 611-626. https://doi.org/10.1037/0012-1649.23.5.611

Bauer, I., Wrosch, C., \& Jobin, J. (2008). I'm better off than most other people: The role of social comparisons for coping with regret in young adulthood and old age. Psychology \& Aging, 23(4), 800-811. http://dx.doi.org/10.1037/a0014180.

Bolger, N., \& Zuckerman, A. (1995). A framework for studying personality in the stress process. Journal of Personality and Social Psychology, 69, 890-902. https://doi.org/10.1037/0022-3514.69.5.890

Brandtstädter, J., \& Renner, G. (1990). Tenacious goal pursuit and flexible goal adjustment: Explication and age-related analysis of assimilative and accommodative strategies of coping. Psychology \& Aging, 5(1), 58-67. http://dx.doi.org/10.1037/0882-7974.5.1.58

Breines, J. G., Thoma, M. V., Gianferante, D., Hanlin, L., Chen, X., \& Rohleder, N. (2014a). 
Self-compassion as a predictor of interleukin-6 response to acute psychosocial stress. Brain, Behavior, \& Immunity, 37, 109-114.

http://dx.doi.org/10.1016/j.bbi.2013.11.006

Breines, J., Thoma, M., Freeman, T., Wu, Z., \& Rohleder, N. (2014b, March). Self-compassion as a predictor of cortisol responses to repeated acute psychosocial stress. Poster presented at the American Psychosomatic Society meeting, San Francisco, CA.

Brion, J. M., Leary, M. R., \& Drabkin, A. S. (2014). Self-compassion and reactions to serious illness: The case of HIV. Journal of Health Psychology, 19(2), 218-229. http://dx.doi.org/10.1177/1359105312467391

Bruce, M. L. (2001). Depression and disability in late life: Directions for future research. American Journal of Geriatric Psychiatry, 9, 102-112. http://dx.doi.org/10.1176/appi.ajgp.9.2.102

Chida, Y., \& Steptoe, A. (2009). Cortisol awakening response and psychosocial factors: a systematic review and meta-analysis. Biological Psychology, 80(3), 265-278. http://dx.doi.org/10.1016/j.biopsycho.2008.10.004

Cohen, S., Janicki-Deverts, D., \& Miller, G. E. (2007). Psychological stress and disease. JAMA, 298(14), 1685-1687. http://dx.doi.org/10.1001/jama.298.14.1685

Cohen, S., Schwartz, J. E., Epel, E., Kirschbaum, C., Sidney, S., \& Seeman, T. (2006). Socioeconomic status, race, and diurnal cortisol decline in the Coronary Artery Risk Development in Young Adults (CARDIA) Study. Psychosomatic Medicine, 68(1), 41-50. http://dx.doi.org/10.1097/01.psy.0000195967.51768.ea

Ebner, N. C., Freund, A. M., \& Baltes, P. B. (2006). Developmental changes in personal goal orientation from young to late adulthood: from striving for gains to maintenance and prevention of losses. Psychology \& Aging, 21(4), 664. http://dx.doi.org/10.1037/0882- 
7974.21.4.664

Festinger, L. (1954). A theory of social comparison processes. Human Relations, 7(2), 117-140. http://dx.doi.org/10.1177/001872675400700202

Folkman, S., Lazarus, R. S., Dunkel-Schetter, C., DeLongis, A., \& Gruen, R. J. (1986).

Dynamics of a stressful encounter: Cognitive appraisal, coping, and encounter outcomes. Journal of Personality and Social Psychology, 50(5), 992-1003. http://dx.doi.org/10.1037/0022-3514.50.5.992

Friis, A. M., Johnson, M. H., Cutfield, R. G., \& Consedine, N. S. (2015). Does kindness matter? Self compassion buffers the negative impact of diabetes distress on HbA1c. Diabetic Medicine, 32(12), 1634-1640. http://dx.doi.org/10.1111/dme.12774

Geiger, P. J., Boggero, I. A., Brake, C. A., Caldera, C. A., Combs, H. L., Peters, J. R., \& Baer, R. A. (2016). Mindfulness-based interventions for older adults: a review of the effects on physical and emotional well-being. Mindfulness, 7(2), 296-307. http://dx.doi.org/10.1007/s12671-015-0444-1

Gilovich, T., Medvec, V. H., \& Kahneman, D. (1998). Varieties of regret: A debate and partial resolution. Psychological Review, 105(3), 602-605. http://dx.doi.org/10.1037/0033295X.105.3.602

Hajat, A., Diez-Roux, A., Franklin, T. G., Seeman, T., Shrager, S., Ranjit, N., ... \& Kirschbaum, C. (2010). Socioeconomic and race/ethnic differences in daily salivary cortisol profiles: the multi-ethnic study of atherosclerosis. Psychoneuroendocrinology, 35(6), 932-943. http://dx.doi.org/10.1016/j.psyneuen.2009.12.009

Heaney, J. L., Phillips, A. C., \& Carroll, D. (2012). Ageing, physical function, and the diurnal rhythms of cortisol and dehydroepiandrosterone. Psychoneuroendocrinology, 37(3), 341349. http://dx.doi.org/10.1016/j.psyneuen.2011.07.001 
Heckhausen, J., Dixon, R. A., \& Baltes, P. B. (1989). Gains and losses in development throughout adulthood as perceived by different adult age groups. Developmental Psychology, 25(1), 109. http://dx.doi.org/10.1037/0012-1649.25.1.109

Heckhausen, J., Wrosch, C., \& Schulz, R. (2010). A motivational theory of life-span development. Psychological Review, 117(1), 32-60. http://dx.doi.org/10.1037/a0017668

Heckhausen, J., Wrosch, C., \& Schulz, R. (in press). Agency and motivation in adulthood and old age. Annual Review of Psychology.

Heim, C., Ehlert, U., \& Hellhammer, D. H. (2000). The potential role of hypocortisolism in the pathophysiology of stress-related bodily disorders. Psychoneuroendocrinology, 25(1), 135. http://dx.doi.org/10.1016/S0306-4530(99)00035-9

Homan, K. J. (2016). Self-compassion and psychological well-being in older adults. Journal of Adult Development, 23(2), 111-119. http://dx.doi.org/10.1007/s10804-016-9227-8

Ice, G. H. (2005). Factors influencing cortisol level and slope among community dwelling older adults in Minnesota. Journal of Cross-Cultural Gerontology, 20(2), 91-108. http://dx.doi.org/10.1007/s10823-005-9085-5

Kiecolt-Glaser, J. K., \& Glaser, R. (2001). Stress and immunity: Age enhances the risks. Current Directions in Psychological Science, 10(1), 18-21. http://dx.doi.org/10.1111/14678721.00105

Kirschbaum, C., Prussner, J. C., Stone, A. A., Federenko, I., Gaab, J., Lintz, D., ... \& Hellhammer, D. H. (1995). Persistent high cortisol responses to repeated psychological stress in a subpopulation of healthy men. Psychosomatic Medicine, 57(5), 468-474. http://dx.doi.org/10.1097/00006842-199509000-00009

Kudielka, B. M., Hellhammer, D. H., \& Wüst, S. (2009). Why do we respond so differently? Reviewing determinants of human salivary cortisol responses to 
challenge. Psychoneuroendocrinology, 34(1), 2-18.

http://dx.doi.org/10.1016/j.psyneuen.2008.10.004

Kumari, M., Shipley, M., Stafford, M., \& Kivimaki, M. (2011). Association of diurnal patterns in salivary cortisol with all-cause and cardiovascular mortality: findings from the Whitehall II study. The Journal of Clinical Endocrinology \& Metabolism, 96(5), 1478-1485.

http://dx.doi.org/10.1210/jc.2010-2137

Landman, J. (1987). Regret: A theoretical and conceptual analysis. Journal for the Theory of Social Behaviour, 17(2), 135-160. http://dx.doi.org/10.1111/j.1468-5914.1987.tb00092.x

Lawton, M. P., \& Brody, E. M. (1969). Assessment of older people: Self-maintaining and instrumental activities of daily living. The Gerontologist, 9, 179-186. http://dx.doi.org/10.1093/geront/9.3_Part_1.179

Leary, M. R., Tate, E. B., Adams, C. E., Batts Allen, A., \& Hancock, J. (2007). Self-compassion and reactions to unpleasant self-relevant events: The implications of treating oneself kindly. Journal of Personality and Social Psychology, 92(5), 887-904. http://dx.doi.org/10.1037/0022-3514.92.5.887

Liu, S. Y., Wrosch, C., Miller, G. E., \& Pruessner, J. C. (2014). Self-esteem change and diurnal cortisol secretion in older adulthood. Psychoneuroendocrinology, 41, 111-120. http://dx.doi.org/10.1016/j.psyneuen.2013.12.010

Miller, G. E., Chen, E., \& Zhou, E. S. (2007). If it goes up, must it come down? Chronic stress and the hypothalamic-pituitary-adrenocortical axis in humans. Psychological Bulletin, 133(1), 25-45. http://dx.doi.org/10.1037/0033-2909.133.1.25

Nater, U. M., Hoppmann, C. A., \& Scott, S. B. (2013). Diurnal profiles of salivary cortisol and alpha-amylase change across the adult lifespan: Evidence from repeated daily life assessments. Psychoneuroendocrinology, 38(12), 3167-3171. 
http://dx.doi.org/10.1016/j.psyneuen.2013.09.008

Neff, K. (2003a). Self-compassion: An alternative conceptualization of a healthy attitude toward oneself. Self \& Identity, 2(2), 85-101. http://dx.doi.org/10.1080/15298860309032

Neff, K. D. (2003b). The development and validation of a scale to measure self-compassion. Self \& Identity, 2(3), 223-250. http://dx.doi.org/10.1080/15298860309027

Neff, K. D. (2011). Self-compassion, self-esteem, and well-being. Social and Personality Psychology Compass, 5(1), 1-12. http://dx.doi.org/10.1111/j.1751-9004.2010.00330.x

Nicolson, N., Storms, C., Ponds, R., \& Sulon, J. (1997). Salivary cortisol levels and stress reactivity in human aging. The Journals of Gerontology Series A: Biological Sciences and Medical Sciences, 52(2), M68-M75. http://dx.doi.org/10.1093/gerona/52A.2.M68

O’Donnell, K., Badrick, E., Kumari, M., \& Steptoe, A. (2008). Psychological coping styles and cortisol over the day in healthy older adults. Psychoneuroendocrinology, 33(5), 601-611. https://doi.org/10.1016/j.psyneuen.2008.01.015

Otte, C., Hart, S., Neylan, T. C., Marmar, C. R., Yaffe, K., \& Mohr, D. C. (2005). A metaanalysis of cortisol response to challenge in human aging: Importance of gender. Psychoneuroendocrinology, 30(1), 80-91. http://dx.doi.org/10.1016/j.psyneuen.2004.06.002

Pruessner, J. C., Kirschbaum, C., Meinlschmid, G., \& Hellhammer, D. H. (2003). Two formulas for computation of the area under the curve represent measures of total hormone concentration versus time-dependent change. Psychoneuroendocrinology, 28, 916-931. http://dx.doi.org/10.1016/S0306-4530(02)00108-7

Raes, F. (2011). The effect of self-compassion on the development of depression symptoms in a non-clinical sample. Mindfulness, 2(1), 33-36. doi: 10.1007/s12671-011-0040-y

Raes, F., Pommier, E., Neff, K. D., \& Van Gucht, D. (2011). Construction and factorial 
validation of a short form of the Self-Compassion Scale. Clinical Psychology \& Psychotherapy. 18, 250-255. http://dx.doi.org/10.1002/cpp.702

Rimmele, U., Zellweger, B. C., Marti, B., Seiler, R., Mohiyeddini, C., Ehlert, U., \& Heinrichs, M. (2007). Trained men show lower cortisol, heart rate and psychological responses to psychosocial stress compared with untrained men. Psychoneuroendocrinology, 32(6), $627-$ 635. http://dx.doi.org/10.1016/j.psyneuen.2007.04.005

Rohleder, N., Kudielka, B. M., Hellhammer, D. H., Wolf, J. M., \& Kirschbaum, C. (2002). Age and sex steroid-related changes in glucocorticoid sensitivity of pro-inflammatory cytokine production after psychosocial stress. Journal of Neuroimmunology, 126(1), 6977.https://doi.org/10.1016/S0165-5728(02)00062-0

Sapolsky, R. M., Krey, L. C., \& McEwen, B. S. (1986). The neuroendocrinology of stress and aging: The glucocorticoid cascade hypothesis. Endocrine Reviews, 7, 284-301. http://dx.doi.org/ 10.1210/edrv-7-3-284

Seeman, T. E., Berkman, L. F., Blazer, D., \& Rowe, J. W. (1994). Social ties and support and neuroendocrine function: The MacArthur studies of successful aging. Annals of Behavioral Medicine, 16(2), 95-106. http://dx.doi.org/10.1093/abm/16.2.95

Segerstrom, S. C., Boggero, I. A., Smith, G. T., \& Sephton, S. E. (2014). Variability and reliability of diurnal cortisol in younger and older adults: implications for design decisions. Psychoneuroendocrinology, 49, 299309. http://dx.doi.org/10.1016/j.psyneuen.2014.07.022

Sephton, S. E., Sapolsky, R. M., Kraemer, H. C., \& Spiegel, D. (2000). Diurnal cortisol rhythm as a predictor of breast cancer survival. Journal of the National Cancer Institute, 92(12), 994-1000. https://doi.org/10.1093/jnci/92.12.994

Tabachnik, B. G., Fidell, L. S., \& Osterlind, S. J. (2013). Using multivariate statistics (6 ${ }^{\text {th }}$ ed.). 
Boston, MA: Pearson.

United Nations, Department of Economic and Social Affairs, Population Division (2015). World Population Ageing 2015 (ST/ESA/SER.A/390).

Van Cauter, E., Leproult, R., \& Kupfer, D. J. (1996). Effects of gender and age on the levels and circadian rhythmicity of plasma cortisol. The Journal of Clinical Endocrinology \& Metabolism, 81(7), 2468-2473. http://dx.doi.org/10.1210/jcem.81.7.8675562

Van Eck, M., Berkhof, H., Nicolson, N., \& Sulon, J. (1996). The effects of perceived stress, traits, mood states, and stressful daily events on salivary cortisol. Psychosomatic Medicine, 58(5), 447-458. http://dx.doi.org/10.1097/00006842-199609000-00007

Vedhara, K., Miles, J. N., Sanderman, R., \& Ranchor, A. V. (2006). Psychosocial factors associated with indices of cortisol production in women with breast cancer and controls. Psychoneuroendocrinology, 31(3), 299-311.

http://dx.doi.org/10.1016/j.psyneuen.2005.08.006

Wrosch, C., \& Heckhausen, J. (2002). Perceived control of life regrets: Good for young and bad for old adults. Psychology \& Aging, 17(2), 340. http://dx.doi.org/10.1037/08827974.17.2.340

Wrosch, C., Bauer, I., \& Scheier, M. F. (2005). Regret and quality of life across the adult life span: the influence of disengagement and available future goals. Psychology \& Aging, 20(4), 657-670. http://dx.doi.org/10.1037/0882-7974.20.4.657

Wrosch, C., Bauer, I., Miller, G. E., \& Lupien, S. (2007). Regret intensity, diurnal cortisol secretion, and physical health in older individuals: Evidence for directional effects and protective factors. Psychology \& Aging, 22(2), 319-330. http://dx.doi.org/10.1037/08827974.22.2.319

Wrosch, C., Dunne, E., Scheier, M. F., \& Schulz, R. (2006). Self-regulation of common age- 
related challenges: Benefits for older adults' psychological and physical health. Journal of Behavioral Medicine, 29(3), 299-306. http://dx.doi.org/10.1007/s10865-006-9051-x

Wrosch, C., Schulz, R., Miller, G. E., Lupien, S., \& Dunne, E. (2007). Physical health problems, depressive mood, and cortisol secretion in old age: Buffer effects of health engagement control strategies. Health Psychology, 26, 341-349. https://doi.org/10.1037/02786133.26 .3 .341

Zhang, J. W., \& Chen, S. (2016). Self-compassion promotes personal improvement from regret experiences via acceptance. Personality and Social Psychology Bulletin, 42(2), 244-258. http://dx.doi.org/10.1177/0146167215623271 


\section{Footnotes}

${ }^{1}$ We note that our study also included a measure of daily perceived stress, which was assessed on three days and could involve both transient and addressable stressors or chronic and uncontrollable stressors. Since our theoretical approach focused on specific chronic and uncontrollable stressors, daily perceptions of stress were not considered for the study hypotheses. However, we acknowledge that supplemental analyses, using an average score of daily perceived stress, did not show significant interactions involving daily stress and self-compassion predicting cortisol level $(\beta=-.22, p>.05)$ or slope $(\beta=-.00, p>.05)$. In addition, there were no significant main effects of the daily stress predicting cortisol level $(\beta=.10, p>.05)$ or cortisol slope $(\beta=$ $.00, p>.05)$. Further, including daily stress into the reported models as a covariate did not change any of the reported results. 

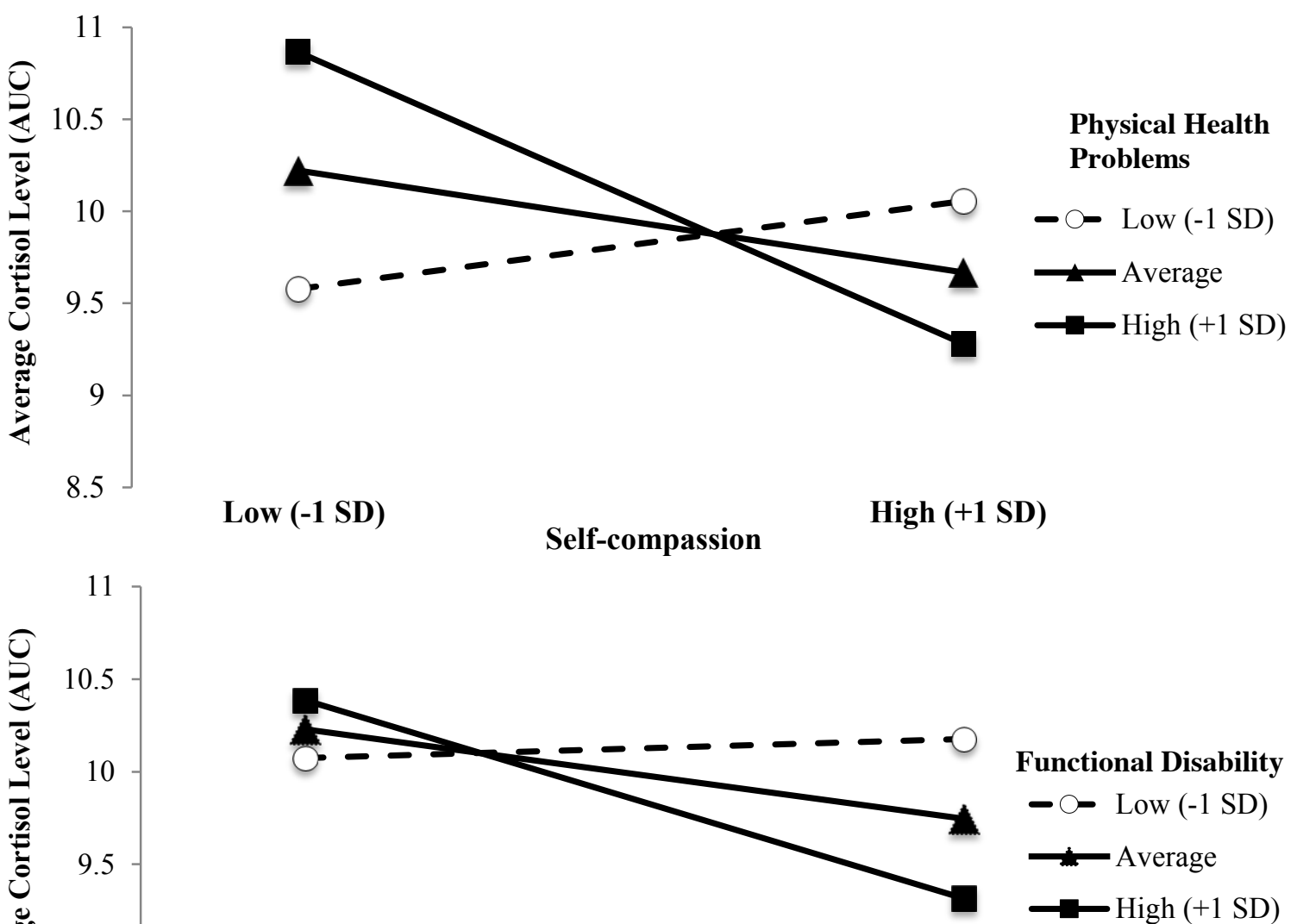

Functional Disability<smiles>CO[Te][AsH2]</smiles>

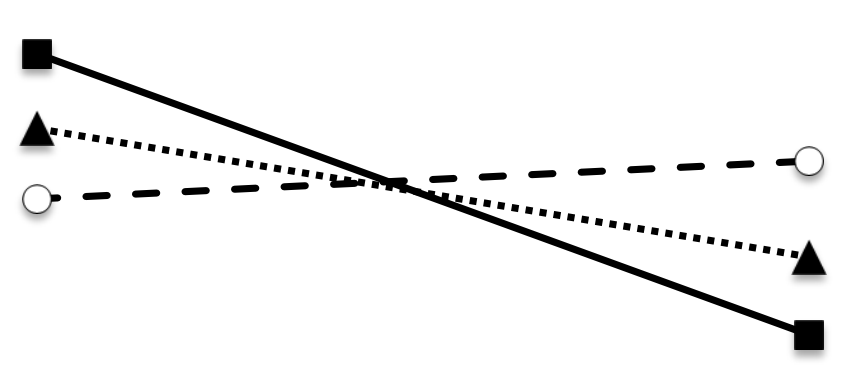

Regret Intensity

Low (-1 SD)

\section{Self-compassion}

High (+1 SD)

Figure 1. Associations between self-compassion and cortisol levels separately for: (upper panel) participants who reported levels of physical health problems one standard deviation above, below and mean levels of physical health problems, (middle panel) levels of functional disability one standard deviation above, below and mean levels of functional disability, and (lower panel) levels of regret emotions one standard deviation above, below and mean levels of regret emotions. 
Table 1

Means, Standard Deviations and Frequencies of Main Study Variables $(N=233)$

\begin{tabular}{lc}
\hline Constructs & Mean (SD) or Percentage \\
\hline Cortisol level (AUC in log nmol/Lxh) & $10.00(2.46)$ \\
Cortisol slope & $-0.04(.01)$ \\
Self-compassion & $41.14(6.33)$ \\
Regret intensity & $4.76(5.10)$ \\
Physical health problems & $1.24(1.12)$ \\
Functional disability & $1.71(2.20)$ \\
Education $(\%)$ & \\
$\quad$ Did not complete high school & 15.02 \\
High school & 21.89 \\
College/trade & 13.30 \\
Bachelor & 25.75 \\
Masters/PhD & 16.74 \\
Income $(\%)$ & \\
Less than $\$ 17,000$ & 7.30 \\
$\$ 17,001-\$ 34,000$ & 26.18 \\
$\quad \$ 34,001-\$ 51,000$ & 21.46 \\
$\quad \$ 51,001-\$ 68,000$ & 9.44 \\
$\quad \$ 68,000-85,000$ & 9.01 \\
$>\$ 85,000$ & 16.31 \\
Perceived social status & $6.69(1.76)$ \\
Married or cohabitating $(\%)$ & 50.21 \\
Age & $75.57(7.75)$ \\
Female $(\%)$ & 60.94 \\
BMI & $26.80(4.78)$ \\
Current smoker $(\%)$ & 4.72 \\
\hline Note that $\%$ ) & \\
\hline
\end{tabular}

Note that that the sample size involving correlations with regret intensity, perceived social status, and $\mathrm{BMI}$ and were slightly reduced because of missing data for these constructs. 
Table 2

Zero-Order Correlations of Main Study Variables $(N=233)$.

\begin{tabular}{lllllllllll}
\hline & 1 & 2 & 3 & 4 & 5 & 6 & 7 & 8 & 9 & 10
\end{tabular}

1. Cortisol level (AUC)

2. Cortisol slope

.12

3. Female

$-.23^{* *} \quad-.06$

4. Age

$.15^{*} \quad .20^{* *} \quad-.06$

5. BMI

$\begin{array}{llll}.05 & .05 & -.07 & -.02\end{array}$

6. Smoking

7. SES

8. Self-compassion

$\begin{array}{lllll}.09 & -.04 & .01 & -.16^{*} & -.13\end{array}$

$\begin{array}{llllll}-.05 & -.03 & -.19^{* *} & -.19^{* *} & -.07 & -.10\end{array}$

9. Physical health problems

10. Functional disability

$\begin{array}{lllllll}-.14^{*} & .00 & .04 & .03 & .02 & .01 & .18^{* *}\end{array}$

$\begin{array}{lllllllll}.08 & .03 & .14^{*} & .08 & -.03 & .02 & -.08 & -.12\end{array}$

$\begin{array}{llllllll}.08 & .03 & .14 & .08 & -.03 & .02 & -.08 & -.12\end{array}$

$\begin{array}{lllllllll}.00 & .12 & .17^{* *} & .28^{* *} & .07 & -.03 & -.23^{* *} & -.06\end{array}$

11. Regret intensity

$\begin{array}{llllllllll}.09 & .09 & .02 & .07 & .07 & .00 & -.02 & -.16^{*} & -.36^{* *} & .12\end{array}$

.08

Note that that the sample size involving correlations with BMI and regret intensity and were slightly reduced because of missing data for these constructs.

${ }^{*} p \leq .05 ;{ }^{* *} p \leq .01$. 
Table 3

Regression analysis predicting cortisol level (AUC) and slope by covariates, self-compassion, physical health problems, functional disability, and regret emotions $(N=233)$.

\begin{tabular}{|c|c|c|c|c|c|c|}
\hline & \multicolumn{3}{|c|}{ Cortisol level (AUC) } & \multicolumn{3}{|c|}{ Cortisol slope } \\
\hline & $R^{2}$ & $\beta$ & SE & $R^{2}$ & $\beta$ & SE \\
\hline \multicolumn{7}{|l|}{ Main Effects } \\
\hline Female & .043 & $-.536^{* *}$ & .163 & .003 & -.001 & .001 \\
\hline Age & .022 & $.398^{*}$ & .169 & .028 & $.003^{* *}$ & .001 \\
\hline BMI & .003 & .136 & .161 & .015 & .002 & .001 \\
\hline Smoking & .013 & .288 & .161 & .000 & .000 & .001 \\
\hline SES & .000 & -.052 & .170 & .001 & .001 & .001 \\
\hline Self-compassion (SC) & .010 & -.273 & .169 & .001 & .001 & .001 \\
\hline Physical health problems & .007 & .230 & .168 & .000 & .000 & .001 \\
\hline Functional disability & .002 & -.127 & .177 & .005 & .001 & .001 \\
\hline Regret intensity & .001 & .088 & .171 & .012 & .002 & .001 \\
\hline \multicolumn{7}{|l|}{ Interactions } \\
\hline Physical health problems X SC & .045 & $-.516^{* *}$ & .150 & .000 & .000 & .001 \\
\hline Functional disability X SC & .015 & $-.293^{*}$ & .152 & .001 & .000 & .001 \\
\hline Regret intensity X SC & .021 & $-.438^{*}$ & .188 & .011 & -.002 & .001 \\
\hline
\end{tabular}

Notes. $R^{2}$ values represent the unique proportion of variance explained in each step of the analyses. $\beta$ represents standardized regression values in each step of the analyses. All interaction terms were tested in separate models

$* p \leq .05 * * p \leq .01$ 